\title{
RECURSOS HUMANOS EM VIGILÂNCIA SANITÁRIA: UMA DISCUSSÃO SOBRE PERFIL PROFISSIONAL
}

\author{
Maria Jalila Vieira de Figueirêdo Leite \\ Cirurgiã-dentista, Mestre em Odontologia Social, Servidora do Centro de Formação de \\ Pessoal/SESAP-RN. \\ jalilaleite@rn.gov.br
}

\section{Angelo Giuseppe Roncalli da Costa Oliveira}

Cirurgião-dentista, Doutor em Odontologia Preventiva e Social, Professor da UFRN. roncalli@terra.com.br

\section{RESUMO}

Este estudo buscou analisar o perfil dos profissionais que atuam na Vigilância Sanitária do Rio Grande do Norte/Brasil. A coleta de dados constou da aplicação de um questionário de pesquisa, aplicado junto aos profissionais que atuam nas Vigilâncias Sanitárias das Secretarias Municipais de Saúde de Natal/RN e Mossoró/RN e da Secretaria Estadual de Saúde/RN. Foi possível concluir que a formação específica em Vigilância Sanitária dos profissionais que atuam no RN, além de ser escassa, demonstra qualidade questionável, já que não há uma regularidade na oferta e não abrange todos os profissionais, concentrandose especialmente nos profissionais que atuam no nível central. Por outro lado, como ponto positivo, constatou-se bons níveis de experiência em serviço, o que pode favorecer a uma boa atuação profissional. O estudo alerta em relação à necessidade de uma maior intervenção nessa área, buscando a superação desses problemas.

PALAVRAS-CHAVES: Vigilância Sanitária, Profissionais de Saúde, Qualificação Profissional

\section{PROFESSIONALS IN HEALTH SYSTEM: ONE STUDY ABOUT THEIR PROFILE}

\begin{abstract}
The aim of this study was analyze the profile of the professionals that work in crossinfection control policies in Rio Grande do Norte/Brazil. The methodology adopted was a quantitative study making use of a questionnaire. The respondents indicated that there is not enough trainning in the area for the professionals, as well as, there is a lack in the quality of this training, given that it's offer is not regular and don't reach all the professionals, but most specifically those who work in THE central health system. On the other hand, the professionals demonstrated having good levels of experience in work, which may favor. A good professional's performance. This study alerts for the need of an intervention in this area in order to overcome difficulties.
\end{abstract}

KEY-WORDS: Professionals of Health System, Professional Qualification 


\section{RECURSOS HUMANOS EM VIGILÂNCIA SANITÁRIA: UMA DISCUSSÃO SOBRE PERFIL PROFISSIONAL}

\section{INTRODUÇÃO}

Os recursos humanos em vigilância sanitária (VISA) acompanham toda a problemática dos recursos humanos do Sistema Único de Saúde. Convive-se com insuficiências qualitativas quanto à disponibilidade de pessoal e acentuadas distorções nas distribuições geográficas, sociais e institucionais desses recursos humanos.

As ações de vigilância sanitária se destacam dentre as demais ações de saúde, por necessitar de uma força de trabalho extremamente qualificada. Seu objeto de atuação tem um caráter complexo, tornando necessária a aquisição de conhecimentos advindos de diversas áreas, justificando também o envolvimento de equipes multidisciplinares (NASCIMENTO, 2001).

A especificidade requerida para uma atuação adequada desse profissional o coloca em uma situação de extrema responsabilidade, na medida em que, considerando o fim principal da vigilância sanitária de favorecer a promoção da saúde da população, muitas vezes para que esse objetivo seja atingido, o profissional da VISA precisa interferir no modo de produção econômico-social.

Por outro lado, necessidades de conhecimento técnico/científico também se colocam como fundamentais, na medida em que, ao lidar com o mercado e tendo que supervisioná-lo, necessário se faz dominar as técnicas e os conhecimentos que o sustentam. Fala-se que a vigilância deve trabalhar fundamentada em um instrumental mais técnico-científico e menos político e improvisado (TEMA, 2002).

Para que esse profissional possa realizar essas ações, é dado aos órgãos da vigilância sanitária o poder de polícia. Nesse caso o Estado intervém, através de seus órgãos e agentes com normas e regulamentos, disciplinando e restringindo, em favor do direito público adequado, os direitos e liberdades individuais (FANUCK, 1987), (MEIRELLES, 2002).

Essa necessidade fez surgir uma prática vertical, unilateral e autoritária, onde a autoridade sanitária exerce seu poder de polícia através da coerção e da sanção. Por outro lado, entende-se atualmente, a necessidade de municiar a sociedade de toda a informação que lhe torne hábil o exercício de seus direitos, em uma parceria indissociável, com o exercício do poder de polícia (FANUCK, 1987).

Documentos institucionais apontam claramente a baixa capacitação dos profissionais que atuam na Vigilância Sanitária, sugerindo problemas quanto à sua forma de atuação, o que poderá resultar em debilidades quanto ao sistema de atenção à saúde (ANVISA, 2001).

Assim, os recursos humanos que atuam na Vigilância Sanitária necessitam ser parte de uma política de desenvolvimento e qualificação profissional, associada a mecanismos de valorização, tais como o plano de cargos, carreiras e salários, em virtude das características particulares do processo de trabalho em Vigilância Sanitária que impõe limitações quanto ao vínculo empregatício, impedimentos quanto ao exercício profissional no setor regulado, 
vulnerabilidade a pressões de ordem política e econômica e não raramente, agressões à integridade física dos profissionais (NASCIMENTO, 2001).

Essa política de desenvolvimento e qualificação profissional deve levar em conta, além dos aspectos referentes a uma formação geral, as necessidades específicas de cada Estado e de cada Município, em função das distintas realidades existentes.

Os problemas de recursos humanos nesta área nunca foram enfrentados de forma organizada: algumas iniciativas vêm ocorrendo, mas têm sido isoladas e sem continuidade. De um modo geral os processos de capacitação profissional são fragmentados, insuficientes, desconectados dos serviços e processos de trabalho dos agentes da Vigilância Sanitária (ANVISA, 2001).

Na tentativa de elucidar algumas dessas questões, este estudo pretendeu conhecer e analisar o perfil profissional da equipe que integra a Vigilância Sanitária no Rio Grande do Norte e sua influência na prática profissional, diferenciando-os quanto ao nível de atuação.

\section{METODOLOGIA}

Esta pesquisa se aproximou ao método quantitativo-descritivo, especificamente sendo um estudo de descrição de população que possui como função primordial a exata descrição de certas características quantitativas de populações como um todo, organizações ou outras coletividades específicas (LAKATOS, 1991).

O Estado do Rio Grande do Norte conta com 133 profissionais atuando na vigilância sanitária, inseridos em níveis de organização estadual, regional, municipal e distrital. Vinte e Nove profissionais são vinculados à Subcoordenadoria de Vigilância Sanitária da Secretaria de Estado da Saúde Pública, 23 profissionais são vinculados às Unidades Regionais de Saúde Pública / Secretaria de Estado da Saúde Pública, 07 profissionais são vinculados à Secretaria Municipal de Saúde de Mossoró / RN e 74 profissionais são vinculados à Secretaria Municipal de Saúde do município de Natal / RN.

Estes profissionais constituíram a nossa população-alvo que teve como fonte de dados a aplicação de um questionário, elaborado em função dos objetivos da pesquisa, com perguntas abertas e fechadas, onde as seguintes informações foram obtidas: local de atuação, sexo, idade, escolaridade, experiências profissionais e de participação em cursos de atualização na área de Vigilância Sanitária. Esse questionário foi aplicado no segundo semestre do ano de 2006, preenchido pelo próprio respondente e devolvido ao pesquisador em um prazo estipulado.

Em se tratando de uma pesquisa com envolvimento de seres humanos, este estudo observou critérios pertinentes à bioética, tais como: preservação do sujeito da pesquisa, autonomia e eqüidade. Assim, exigências fundamentais, tais como o termo de consentimento livre e esclarecido dos indivíduos e a não identificação dos pesquisados foram consideradas, a priori, como compromissos primordiais do pesquisador.

A pesquisa constou das seguintes etapas: 
1. Estudo exploratório - Identificação dos recursos humanos que atuam nos serviços de vigilância sanitária estadual e dos municípios de Natal e Mossoró/RN e elaboração do instrumento da pesquisa.

2. Estudo Piloto - Pré-teste do questionário, objetivando corrigir as distorções apresentadas, identificar se o mesmo estava de fato aplicável e se respondia efetivamente aos problemas colocados pelo investigador. Em função desses resultados e de sua análise em um banco de dados, a versão definitiva do questionário foi obtida.

3. Coleta de dados - Os questionários foram enviados através de correio e/ou entregues pessoalmente aos responsáveis pelo setor de vigilância sanitária das respectivas Instituições. Na data agendada foi solicitada a devolução do material. Um re-envio foi realizado com o objetivo de melhorar a taxa de resposta.

4. Análise e Interpretação dos dados, à luz do referencial teórico, com base nos objetivos da pesquisa.

\section{RESULTADOS E DISCUSSÃO}

Na primeira devolução dos questionários obteve-se um percentual de resposta de 45,4 \% (64 formulários). Após o segundo envio, obteve-se o número final de 76 respondentes, o que corresponde a 57,14\% do número total (Tabela 01).

A taxa de não-resposta foi mais baixa entre os técnicos que atuam no nível central da Secretaria de Estado da Saúde Pública (SESAP) e no nível distrital da Secretaria Municipal de Saúde (SMS) de Natal. Segundo informações coletadas junto aos responsáveis, vários fatores podem ter contribuído para este resultado: técnicos em férias ou afastados do serviço, por licença temporária; viagens freqüentes e visitas de campo dos técnicos; excesso de atividades e não relação direta da pesquisa com o seu trabalho no setor; não gostar de preencher questionários; não disponibilidade imediata de alguns dados solicitados e pouca valorização da importância de pesquisas científicas nos serviços de saúde.

\begin{tabular}{c|c|cc}
$\begin{array}{c}\text { Tabela 01 - Número de profissionais que participaram da } \\
\text { pesquisa, em relação ao local de atuação. RN, 2003 }\end{array}$ \\
\hline $\begin{array}{c}\text { Instituição/No } \\
\text { Profissionais }\end{array}$ & $\begin{array}{c}\text { Profissionais } \\
\text { lotados* }\end{array}$ & $\begin{array}{c}\text { Profissionais que } \\
\text { responderam o } \\
\text { formulário } \\
\text { \% }\end{array}$ \\
\hline SESAP Central & & n & 51,72 \\
SESAP Regionais & 29 & 15 & 78,26 \\
SMS Mossoró & 07 & 18 & 100,00 \\
SMS Natal Central & 15 & 11 & 73,33 \\
SMS Natal Distritos & 59 & 25 & 42,37 \\
\hline Total & $\mathbf{1 3 3}$ & $\mathbf{7 6}$ & $\mathbf{5 7 , 1 4}$ \\
\hline
\end{tabular}

*Foram excluídos da listagem profissionais que se envolviam apenas em ações burocráticas e administrativas

\section{Sexo e Idade}

Os profissionais pesquisados são predominantemente do sexo feminino. Estes dados estão em acordo com o trabalho de ARAÚJO, GUERRA (2001), que encontrou um percentual 
de $74,1 \%$ dos profissionais pesquisados, do sexo feminino. Os profissionais pesquisados têm, em média, 41 anos. Verifica-se uma menor homogeneidade entre os pesquisados da SMS Mossoró, SESAP Regionais e SMS Natal Central que apresentam maiores desviospadrões, indicando equipes com pessoas de idades mais diferentes.

ARAÚJO, GUERRA (2001) encontraram 53,4 \% dos pesquisados entre 30 a 39 anos e $37,9 \%$ de 40 a 49 anos.

Tabela 02 - Distribuição dos profissionais em relação ao sexo, RN, 2003

\begin{tabular}{l|cc|cc|cc}
\hline \multicolumn{1}{c|}{$\begin{array}{c}\text { Local de } \\
\text { Atuação/Sexo }\end{array}$} & \multicolumn{2}{c|}{ Masculino } & \multicolumn{2}{c|}{ Feminino } & \multicolumn{2}{c}{ Total } \\
& $\mathbf{n}$ & $\mathbf{\%}$ & $\mathbf{n}$ & $\mathbf{\%}$ & \multicolumn{2}{|c}{$\mathbf{n}$} \\
\% & & & & & & \\
\hline SESAP Central & 05 & 33,33 & 10 & 66,66 & 15 & 100,00 \\
SESAP Regionais & 10 & 55,55 & 08 & 44,44 & 18 & 100,00 \\
SMS Mossoró & 04 & 57,14 & 03 & 42,86 & 07 & 100,00 \\
SMS Natal Central & 03 & 27,27 & 08 & 72,73 & 11 & 100,00 \\
SMS Natal Distritos & 09 & 36,00 & 16 & 64,00 & 25 & 100,00 \\
\hline \multicolumn{1}{c|}{ Total } & $\mathbf{3 1}$ & $\mathbf{4 0 , 7 9}$ & $\mathbf{4 5}$ & $\mathbf{5 9 , 2 1}$ & $\mathbf{7 6}$ & $\mathbf{1 0 0 , 0 0}$ \\
\hline
\end{tabular}

Tabela 03 - Média de idade dos profissionais, em relação ao local de atuação, RN, 2003

\begin{tabular}{l|c|c}
\hline \multicolumn{1}{c|}{ Local / Idade } & Média & Desvio-Padrão \\
\hline SESAP Central & 43,4 & 5,27 \\
SESAP Regionais & 44,5 & 8,79 \\
SMS Mossoró & 35,6 & 10,4 \\
SMS Natal Central & 43,1 & 8,17 \\
SMS Natal Distritos & 38,7 & 4,89 \\
\hline
\end{tabular}

\section{Escolaridade}

Neste estudo, dentre os respondentes, 14 profissionais têm formação em ensino médio, onde 12 deles têm formação técnica na área de vigilância sanitária, correspondendo a 15,8 \% do total de pesquisados. Há uma predominância destes profissionais na SMS Natal, com ausência dos mesmos na SESAP Central.

FEKETE (2001) relata que, em relação ao nível médio, o setor saúde vem historicamente incorporando pessoal sem qualificação para atuar nas distintas áreas, incluindo Vigilância Sanitária. Em pesquisa realizada de abrangência nacional, a mesma encontrou que os profissionais de nível médio nos serviços estaduais somam 1.208, sendo a maior parte sem formação específica na área de saúde ou de VISA. No entanto, essa realidade não foi constatada neste estudo, onde se observa que dentre o total de profissionais de nível médio, 85,7\% tem formação técnica específica em VISA.

Dentre os 62 profissionais que relataram ter cursos de formação superior (84,2\%), 05 categorias estão mais presentes: os farmacêuticos (21\%); os enfermeiros (16,1\%); os cirurgiões-dentistas $(12,9 \%)$; os médicos-veterinários $(11,3 \%)$ e os nutricionistas $(8,1)$ (Tabela 04). 
Tabela 04 - Número de Profissionais por área de graduação, em relação ao local de atuação, RN, 2003

\begin{tabular}{l|c|c|c|c|c|c}
\hline $\begin{array}{l}\text { Graduação/ Local } \\
\text { de Atuação }\end{array}$ & $\begin{array}{c}\text { SESAP } \\
\text { Central }\end{array}$ & $\begin{array}{c}\text { SESAP } \\
\text { Regionais }\end{array}$ & $\begin{array}{c}\text { SMS } \\
\text { Mossoró }\end{array}$ & $\begin{array}{c}\text { SMS } \\
\text { Natal } \\
\text { Central }\end{array}$ & $\begin{array}{c}\text { SMS } \\
\text { Natal } \\
\text { Distritos }\end{array}$ & Total \\
\hline Farmácia & 02 & 04 & 01 & 02 & 04 & 13 \\
Medicina- & 01 & 04 & 02 & 0 & 0 & 07 \\
Veterinária & 03 & 01 & 01 & 01 & 04 & 10 \\
Enfermagem & 01 & 0 & 0 & 0 & 0 & 01 \\
Direito & 03 & 0 & 01 & 01 & 03 & 08 \\
Odontologia & 0 & 0 & 01 & 01 & 02 & 04 \\
Ciências Biológicas & 0 & 0 & 0 & 02 & 0 & 02 \\
Psicologia & 02 & 0 & 0 & 01 & 02 & 05 \\
Nutrição & 0 & 0 & 0 & 01 & 02 & 03 \\
Educação Física & 0 & 0 & 0 & 0 & 01 & 01 \\
Fisioterapia & 02 & 04 & 0 & 0 & 02 & 08 \\
Outras* & $\mathbf{1 4}$ & $\mathbf{1 3}$ & $\mathbf{0 6}$ & $\mathbf{0 9}$ & $\mathbf{2 0}$ & $\mathbf{6 2}$ \\
\hline Total & & 0 & 02 & \\
\hline
\end{tabular}

*Ciências Contábeis, Serviço Social, Pedagogia, Ciências Econômicas, Comunicação Social, Analista de Sistema, Letras

**Apenas 03 respondentes fizeram um $2^{\circ}$ curso de graduação, nas áreas de Enfermagem, Administração e Ciências Biológicas

Alguns aspectos observados em relação às categorias profissionais merecem uma referência especial:

- Inexistência de médico-veterinários na SMS Natal, sendo mais presentes no interior do Estado (SESAP Regionais e SMS Mossoró);

- Presença de advogado apenas na SESAP Central. Esta situação ainda é boa quando comparada aos resultados obtidos por LUCCHESE (2001) em pesquisa realizada junto a 18 Estados, onde em nenhum deles existia assessoria jurídica específica para a Vigilância Sanitária;

- Inexistência de cirurgiões-dentistas nas Regionais da SESAP;

- Inexistência de médicos atuando na Vigilância Sanitária do RN; situação adversa à nacional, onde se encontra um percentual considerável destes profissionais;

- Grande diversidade de categorias profissionais na SMS Natal;

- Presença de profissionais com cursos não afins à área de vigilância sanitária, correspondendo a $10,5 \%$ dos pesquisados.

FEKETE (2001) relata que em um Levantamento da Situação dos Serviços de Vigilância Sanitária no Brasil realizado em 2001 em todos os Estados, Capitais e Municípios com mais de 200.000 habitantes, as categorias mais numerosas são: farmacêuticos (246), enfermeiros (179), médico-veterinários (199), cirurgiões-dentistas (157) e médicos (105), dados muito parecidos ao deste trabalho, com exceção da categoria médica. Nos serviços estaduais pesquisados atuam 3.146 profissionais, sendo 225 de nível elementar (7\%), 1.208 de nível médio (37,8\%) e 1.713 de nível superior (53,6\%) e nos serviços municipais foram encontrados 831 profissionais de nível médio (50,5\%), 167 de nível elementar (0,2\%) e 646 de nível superior (39,3\%).

Os resultados desta pesquisa também estão em acordo com os dados de LUCCHESE (2001) quando, excluindo o Estado de São Paulo (que tem um elevado número de 
cirurgiões-dentistas e assim exercem influência considerável nos resultados percentuais), encontrou como mais freqüente a categoria de farmacêuticos, seguida pelos enfermeiros, médico-veterinários e cirurgiões-dentistas. O mesmo afirma que essa situação é favorável, na medida em que a profissão de farmacêutico tem uma grande afinidade com a vigilância sanitária, em função de sua versatilidade para atuar na área de serviços e também na de produtos.

Uma outra questão abordada por LUCCHESE (2001) foi a grande presença de profissionais de nível superior com formações não relacionadas à vigilância sanitária (30\%). No seu trabalho ele encontrou psicólogos, sociólogos, geógrafos e assistentes sociais trabalhando na área. Nesta pesquisa esses profissionais corresponderam a 12,9\% dos pesquisados. Essa situação é preocupante, na medida em que indica uma pouca racionalidade na política de recursos humanos na área de vigilância sanitária.

ARAÚJO, GUERRA (2001) em pesquisa realizada junto aos profissionais da Vigilância Sanitária de Natal encontraram como profissões mais freqüentes: técnico em saneamento (25,9\%), nutricionista (18,97\%), cirurgião-dentista (13,8\%), farmacêutico (12,1\%) e biólogo e enfermeiro, com 10,3\% cada. Esta situação se diferencia dos resultados nacionais e dos resultados desta pesquisa, quando o farmacêutico aparece em terceira colocação e surpreende pelo alto número de nutricionistas e técnicos em saneamento. Os resultados se aproximam aos deste estudo quando demonstra a diversidade de profissionais em suas equipes e também pelo fato dos técnicos com formação em VISA que participaram desse estudo estarem de uma maneira quase integral, lotados na SMS Natal.

No estudo de ARAÚJO, GUERRA (2001) quando eles excluem os profissionais de nível médio, aparecem como profissões mais freqüentes: nutrição e odontologia (16,3\% cada), biologia e farmacêutico (14,3\% cada) e enfermagem com 12,2\% dos pesquisados.

Nesta pesquisa, os profissionais de nível superior tinham em média 16,0 anos de graduados. De uma forma geral, deduz-se que eles tenham uma boa experiência profissional. A equipe da SMS Mossoró é a mais jovem, com uma média de 11,3 anos de graduados. Os níveis centrais da SESAP e SMS Natal se apresentaram como as equipes com maior tempo de conclusão da graduação entre os seus integrantes $(18,8$ e 19,1 anos respectivamente).

ARAÚJO, GUERRA (2001), encontraram no seu estudo que a maior parte dos profissionais tem entre 15 e 24 anos de formados (46,9\%); em seguida, com 34,7\% estão os que têm entre 05 e 14 anos de formados; com até 04 anos tem-se 8,2\%; e apenas 2,0 \% têm entre 25 e 34 anos de formados.

Dentre os 62 profissionais de nível superior que informaram este item, 83,9\% concluíram sua graduação em Universidades do Rio Grande do Norte e 16,1\% em outras Universidades Brasileiras.

De uma forma geral, é muito baixo o percentual de pessoas com cursos de pós-graduação. O número total de cursos citados é pequeno (44), sendo a maioria deles cursos de aperfeiçoamento e especialização, com percentuais muito aproximados. Em relação ao local de atuação, observamos a ausência de profissionais com cursos de pós-graduação nas Regionais da SESAP e na SMS Mossoró e uma distribuição equilibrada entre o Nível Central da SESAP e SMS Natal (Tabela 05). 
Tabela 05- Número de cursos de pós-graduação, em relação ao local de atuação, RN, 2003

\begin{tabular}{|c|c|c|c|c|c|c|}
\hline \multirow{2}{*}{$\begin{array}{l}\text { Local de } \\
\text { Atuação / } \\
\text { Curso }\end{array}$} & \multicolumn{2}{|c|}{ Aperfeiçoamento } & \multicolumn{2}{|c|}{ Especialização } & \multirow[t]{2}{*}{ Mestrado } & \multirow[t]{2}{*}{ Doutorado } \\
\hline & VISA & NãoVISA & VISA & Não VISA & & \\
\hline $\begin{array}{l}\text { SESAP } \\
\text { Central }\end{array}$ & 02 & 03 & 06 & 04 & 01 & 0 \\
\hline $\begin{array}{l}\text { SESAP } \\
\text { Regionais }\end{array}$ & 0 & 0 & 0 & 0 & 0 & 0 \\
\hline SMS Mossoró & 0 & 02 & 0 & 03 & 0 & 0 \\
\hline $\begin{array}{l}\text { SMS Natal } \\
\text { Central }\end{array}$ & 04 & 01 & 03 & 03 & 01 & 0 \\
\hline $\begin{array}{l}\text { SMS Natal } \\
\text { Distritos }\end{array}$ & 04 & 05 & 01 & 03 & 0 & 0 \\
\hline Total & 10 & 11 & 10 & 13 & 02 & 0 \\
\hline
\end{tabular}

Comunicação de rádio e TV, Endodontia, Educação física em Atletismo, Prótese Fixa, Análises Clínicas; Educação Ambiental; Direito Processo Cível, Gestão em áreas turísticas, Metodologia Assistência de Enfermagem, Farmácia Hospitalar, Ortodontia, Alimentos e Nutrição, Psicologia Social, Educação Física para portadores de necessidades especiais; Microbiologia de Alimentos; Bioquímica da Nutrição.

Observa-se ainda um alto percentual de cursos de pós-graduação não relacionados à área (11 cursos de aperfeiçoamento e 13 de especialização), o que indica uma possível não dedicação exclusiva à área, ou pelo menos uma busca por atuação em outras áreas (Tabela 05).

FEKETE (2001) relata em seu estudo, que dentre os profissionais de nível superior que atuam em Vigilância Sanitária no Brasil, 219 tem curso de saúde pública (12,8\%), 111 tem especialização em vigilância sanitária (6,5\%), 53 (3\%) são mestres e 04 (0,2\%) são doutores. A mesma reforça que os processos de formação para o nível superior (graduação/pós-graduação) e para o nível médio são insuficientes quanto aos aspectos mais abrangentes ao processo saúde/doença e quanto ao desenvolvimento de competências e habilidades para atuação no campo da vigilância sanitária.

ARAÚJO, GUERRA (2001) encontraram um percentual de 34,7\% dos profissionais que atuam na Vigilância Sanitária do município de Natal, com pós-graduação e 36,2\% dos profissionais que não participaram de nenhum processo de capacitação.

A escassez de processos de capacitação, especialmente em cursos formais, relatada em nossos resultados, está em acordo com diversos autores, havendo inclusive documentos institucionais que apontam a urgência em resolver esse problema com uma maior oferta de cursos, através do estabelecimento de parcerias com Escolas de Saúde Pública e Universidades, contando ainda com a ferramenta do ensino à distância. Além de cursos, há citações quanto à necessidade de incentivar e criar mecanismos para o desenvolvimento de pesquisas, inclusive aquelas desenvolvidas em serviço, voltadas para a temática da vigilância sanitária, através das agências de financiamento (NASCIMENTO, 2001), (FEKETE, 2001), (LUCCHESE, 2001), (ANVISA, 2001). 
Em relação ao tempo de conclusão destes cursos, observou-se que dentre os que fizeram cursos de aperfeiçoamento, há uma média de 6,8 anos de conclusão, já nos cursos de especialização, a média de conclusão sobe para 11,3 anos.

Todos os cursos de aperfeiçoamento foram realizados fora do Estado, sendo 70\% deles na área de Vigilância Sanitária e oferecidos por uma única Instituição, a ENSP / FIOCRUZ. 20\% dos cursos de especialização foram realizados no Estado (a maioria na área de saúde pública) e o restante fora do Estado, em Instituições diversas.

\section{Cursos de Atualização}

Dentre os respondentes, chama atenção o fato de 18 (29,7\%) não ter preenchido este campo do formulário, deduz-se assim, que eles não participaram de cursos de atualização já que as orientações para o preenchimento do formulário esclareciam que qualquer espaço não preenchido seria entendido como não existir, de fato, a informação (Tabela 06).

\begin{tabular}{|c|c|c|c|c|}
\hline $\begin{array}{l}\text { Local / No Profissionais por } \\
n^{0} \text { cursos }\end{array}$ & $\begin{array}{l}\text { Nenhum } \\
\text { curso }\end{array}$ & $\begin{array}{l}1 \text { a } 3 \\
\text { cursos }\end{array}$ & $\begin{array}{c}4 \text { ou } \\
\text { mais } \\
\text { cursos }\end{array}$ & Total \\
\hline SESAP Central & 05 & 02 & 08 & 15 \\
\hline SESAP Regionais & 03 & 12 & 03 & 18 \\
\hline SMS Mossoró & $\ldots$ & 07 & $\ldots$ & 07 \\
\hline SMS Natal Central & 01 & 03 & 07 & 11 \\
\hline SMS Natal Distritos & 09 & 07 & 09 & 25 \\
\hline Total & 18 & 31 & 27 & 76 \\
\hline
\end{tabular}

Observa-se que as pessoas que participaram de um número maior de cursos estão lotadas no Nível Central da SESAP. A SMS Mossoró é um dos locais de atuação em que os funcionários menos participaram de cursos, isso talvez se relacione com o difícil acesso ou ainda com o menor tempo de formado, como foi observado anteriormente (Tabela 07).

\begin{tabular}{l|c}
$\begin{array}{c}\text { Tabela } 07 \text { - Número de cursos de atualização, em relação ao } \\
\text { local de atuação, RN, 2003 }\end{array}$ \\
\hline Local / Total de Cursos & $\mathbf{N}^{\mathbf{0}}$ Cursos \\
\hline SESAP Central & 73 \\
SESAP Regionais & 44 \\
SMS Mossoró & 08 \\
SMS Natal Central & 45 \\
SMS Natal Distritos & 70 \\
\hline Total & $\mathbf{2 4 0}$ \\
\hline
\end{tabular}

Em relação à duração destes cursos, as mais freqüentes foram cursos de 21 a 40 horas, onde se enquadram 56,7\% dos cursos relatados. O segundo maior percentual enquadrou cursos com até 20 horas, que somados à freqüência anterior compõem 81,3\% dos cursos 
(Tabela 08). A carga horária mais freqüente foi 40 horas, com 41,2\%, seguida por cursos com 20 horas (20,3\%) e cursos com 80 horas $(6,9 \%)$.

\begin{tabular}{|c|c|c|c|c|c|}
\hline $\begin{array}{l}\text { Local de Atuação } \\
\text { / No Cursos por } \\
\text { carga horária }\end{array}$ & $\begin{array}{c}\text { Cursos } \\
\text { com até } \\
20 \\
\text { horas }\end{array}$ & $\begin{array}{c}\text { Cursos } \\
\text { de } 21 \text { a } \\
40 \\
\text { horas }\end{array}$ & $\begin{array}{c}\text { Cursos } \\
\text { de } 41 \text { a } \\
60 \\
\text { horas }\end{array}$ & $\begin{array}{c}\text { Cursos } \\
\text { de } 61 \text { a } \\
80 \\
\text { horas }\end{array}$ & $\begin{array}{c}\text { Cursos } \\
\text { com } \\
\text { mais de } \\
80 \\
\text { horas } \\
\end{array}$ \\
\hline SESAP Central & 13 & 30 & 01 & 05 & - \\
\hline SESAP Regionais & 01 & 18 & 01 & 11 & 01 \\
\hline SMS Mossoró & - & 01 & - & 07 & - \\
\hline $\begin{array}{l}\text { SMS Natal } \\
\text { Central }\end{array}$ & 15 & 23 & 01 & 01 & 01 \\
\hline $\begin{array}{c}\text { SMS Natal } \\
\text { Distritos }\end{array}$ & 17 & 34 & 02 & 01 & 03 \\
\hline Total & 46 & 106 & 05 & 25 & 05 \\
\hline
\end{tabular}

* Tivemos 187 cursos com informações quanto à carga horária

Quanto às Instituições que ofereceram esses cursos, a SMS Natal participa com 86 cursos oferecidos, o que corresponde a 35,8\% do total. Em seguida temos a SESAP, com 37 cursos, correspondendo a 15,4\%, seguidas pelo Instituto de Radioproteção e Dosimetria (IRD); Comissão Nacional de Energia Nuclear (CNEN); Centro Regional de Ciências Nucleares (CRCN) com 28 cursos (11,6\%) e Agência Nacional de Vigilância Sanitária (ANVISA) e Ministério da Saúde, com 23 cursos (9,6\%). Além destas, várias outras instituições participaram como patrocinadoras de cursos.

Ao relacionar-se o local de atuação dos técnicos à Instituição patrocinadora, verifica-se que a ANVISA e o Ministério da Saúde oportunizaram cursos principalmente para os técnicos da SESAP, com ênfase para os profissionais que atuam no nível central. O IRD, CNEN e CRCN também priorizaram os técnicos da SESAP, com um baixo percentual de cursos oferecidos para os técnicos da SMS Natal. A SMS Natal tem sido a maior patrocinadora de cursos para seus técnicos, com 86 cursos dentre os 240 citados.

A SESAP também ofereceu cursos, majoritariamente para sua equipe, onde dos 37 cursos que foram promovidos pela mesma, 23 foram relatados por técnicos das URSAPs e 11 por técnicos do nível central.

Esta situação guarda uma relação esperada entre os níveis de gestão. No caso do RN, onde apenas os municípios de Natal e Mossoró assumem integralmente as ações de VISA e ainda cabe ao Estado atuar frente aos demais municípios, não é surpreendente que a equipe estadual necessite realmente dessa educação continuada.

Uma condição particularmente difícil se apresenta na SMS Mossoró, onde um baixíssimo nível de cursos foi relatado pelos pesquisados. Isso demonstra que, se não tiver ocorrido omissão no preenchimento do questionário, esta é a equipe mais carente de capacitação no 
Estado. Isto pode ser explicado pelo fato da equipe ser muito nova e do município ter assumido recentemente as funções de vigilância sanitária.

De uma forma geral, a maior parte dos cursos de atualização foram realizados no RN (151), correspondendo a 70,9\%, destes, 51,2\% foram realizados em Natal. Fora do Estado, a cidade mais citada é Rio de Janeiro, com 20 cursos (0,8\%).

Os técnicos do Nível Central da SESAP foram os que mais relataram participar de cursos fora do Estado (46), quase o dobro dos que foram realizados aqui no Estado. Esta situação é aceitável, na medida em que a VISA Estadual, sendo responsável por um leque de ações mais ampliadas, inclusive de média e alta complexidade, requerem uma formação que não é disponível no âmbito estadual. No entanto, indica-se a necessidade de uma maior descentralização desses cursos para o pessoal que atua nas Regionais da SESAP. A SMS Natal demonstra uma maior organização, enviando técnicos para cursos fora do Estado, situação que não é acompanhada pela SMS Mossoró e que merece uma imediata inversão, considerando-se o porte do município, seus riscos e suas responsabilidades.

Uma questão importante é que este estudo não identificou nenhum curso de atualização ou pós-graduação que tenha como tema central a educação em saúde. Esse resultado surpreende porque a diversidade de saber necessário aos técnicos que atuam em vigilância sanitária é tão grande que, além dos conhecimentos relativos à VISA propriamente ditos, pela alta responsabilidade educadora da VISA, esses técnicos necessitam ser capacitados em abordagens de educação em saúde e outros conhecimentos para um exercício mais adequado do seu papel educador. Autores e documentos institucionais relatam a preocupação dos técnicos necessitarem traduzir os riscos inerentes ao processo produtivo para a comunidade (LIMA, 1987), (BAHIA, 1998).

Ao se analisar os cursos em relação ao período em que foram ofertados, não há uma regularidade - uma condição negativa reforçada por GOMES ET AL (1987) que relatam a necessidade dos fiscais serem treinados periodicamente, devido ao grande avanço tecnológico.

FEKETE (2001) nos possibilita concluir essa discussão quando diz que a disponibilidade de recursos humanos adequadamente preparados é fator indissociável da qualidade e do volume da produção de serviços e que é responsabilidade do gestor promover o desenvolvimento de seus recursos humanos, através de processos de capacitação e formação profissional.

\section{Experiência Profissional}

Dentre os pesquisados, a SESAP foi a instituição que apresentou técnicos com maior tempo de experiência profissional atual (média de 7,96 anos no nível central e 10,02 nas Regionais), essa situação é coerente, já que é a Instituição mais antiga no Estado. Em segundo lugar temos a SMS Natal e por fim a SMS Mossoró que conta com profissionais pouco experientes em seu quadro (Tabela 09). 
Tabela 09 - Experiência profissional atual e anterior, em relação ao local de atuação, RN, 2003

\begin{tabular}{l|c|c}
\hline $\begin{array}{l}\text { Local de Atuação / Média de } \\
\text { Experiência }\end{array}$ & $\begin{array}{c}\text { Média de anos } \\
\text { de Experiência } \\
\text { Atual }\end{array}$ & $\begin{array}{c}\text { Média de anos de } \\
\text { Experiência } \\
\text { Anterior* }\end{array}$ \\
\hline SESAP Central & 7,96 & 2,16 \\
SESAP Regionais & 10,02 & 4,7 \\
SMS Mossoró & 1,5 & 11,0 \\
SMS Natal Central & 5,0 & 5,0 \\
SMS Natal Distritos & 7,16 & 3,5 \\
\hline
\end{tabular}

* $\mathrm{N}^{\circ}$ Pessoas que indicaram Experiência Anterior: SESAP Central - 03; SESAP Regional - 09; SMS Mossoró - 01; SMS Natal Central - 06 e SMS Natal Distritos - 06

O número de técnicos que indicaram experiência anterior foi maior na SESAP e na SMS Natal, no entanto a média de anos de experiência entre os técnicos variou de 11,0 anos na SMS Mossoró e 2,16 na SESAP Central (Tabela 09).

Ao se verificar a situação de experiência profissional, quando consideradas a experiência anterior e atual juntas, identifica-se que os profissionais que atuam na VISA do RN têm uma boa experiência. As situações de experiência atual relatadas nas VISAs de Natal e Mossoró são mais baixas, no entanto esses profissionais já tinham uma experiência anterior em VISA, no nível Estadual. Isso talvez ajude a superar, em parte, a problemática da débil escolaridade e educação continuada (Tabela 09).

\section{CONCLUSÕES}

Os profissionais da vigilância sanitária participantes desta pesquisa são predominantemente jovens, do sexo feminino e com grau de escolaridade universitário. Níveis de pós-graduação são baixos, com predominância quase que integral de cursos de aperfeiçoamento e especialização.

A formação específica em VISA desses profissionais além de ser escassa e irregular na oferta, não abrange todos os profissionais, concentrando-se especialmente nos que atuam no nível central.

Por outro lado, como ponto positivo, constatou-se bons níveis de experiência em serviço, o que pode favorecer a uma boa atuação profissional. O estudo alerta em relação à necessidade de uma maior intervenção no que diz respeito à qualificação profissional nessa área, buscando a superação desses problemas. Deve-se observar, especialmente, a necessária ampliação da qualificação aos profissionais que atuam em todas as regiões do Estado.

\section{REFERÊNCIAS BIBLIOGRÁFICAS}

1. NASCIMENTO, A. Caderno de Debates da $1^{\text {a }}$ Conferência Nacional de Vigilância Sanitária. Rio de Janeiro: FIOCRUZ. Out/Nov, 2001.

2. TEMA - VIGILÂNCIA SANITÁRIA. Rio de Janeiro: FIOCRUZ. Abr/Mai, 2002. 
3. FANUCK,L.C. Justiça na saúde: quem age na defesa do povo? Saúde em Debate. p 12-14, set/out, 1987.

4. MEIRELLES, H.L. Poderes Administrativos. In: MEIRELLES, H.L. atualizado por Azevedo, E.A, Burle Filho, J.E. Direito Administrativo Brasileiro. 27 ed. São Paulo: Malheiros. p.112-143, 2002.

5. AGÊNCIA NACIONAL DE VIGILÂNCIA SANITÁRIA. I Conferência Nacional de Vigilância Sanitária. Relatório Final. Brasília: ANVISA, 2001.

6. LAKATOS, E.V. MARCONI, MA. Técnicas de Pesquisa. In: Lakatos, E.V., Marconi, M.A. Fundamentos de Metodologia Científica. 3ed. São Paulo: Atlas. Cap: Técnicas de Pesquisa, p.174-214, 1991.

7. ARAÚJO, L.U.A., GUERRA, M.S.A. Pesquisa - Perfil dos profissionais de Vigilância Sanitária da Secretaria Municipal de Saúde de Natal/RN. http://www.observatorio.nesc.ufrn.br, 2001.

8. FEKETE, C. A questão dos recursos humanos na Conferência Nacional de Vigilância Sanitária. 10p. http://www.anvisa.gov.br, 2001.

9. LUCCHESE, G. Globalização e regulação sanitária: os rumos da vigilância sanitária no Brasil. Tese de Doutorado. ENSP/FIOCRUZ. Ago, 2001

10. AGÊNCIA NACIONAL DE VIGILÂNCIA SANITÁRIA. Sistema Nacional de Vigilância Sanitária: política de recursos humanos para o SNVS. http://www.anvisa.gov.br.

11. LIMA, L.F.M. Participação popular e vigilância sanitária. Saúde em Debate. Set/out: p.06-08, 1987.

12. BAHIA.SECRETARIA DA SAÚDE DO ESTADO DA BAHIA. Manual de Procedimentos Técnicos em Ações Básicas de Vigilância Sanitária. GRAFPEL: Salvador, 289p, 1998.

13. GOMES, C.R. ET AL. Encontro avalia ações de Vigilância Sanitária. Saúde em Debate. Set/out: p.41-44, 1987. 